\title{
A GLOBAL LOCALIZATION SYSTEM FOR STRUCTURED ENVIRONMENTS USING AN IMAGING SONAR
}

\author{
Guillermo García de Marina, David Ribas, Pere Ridao
}

\author{
Dept. Arquitectura i Tecnologia de Computadors \\ Universitat de Girona \\ Girona, Spain
}

\begin{abstract}
This paper describes a localization system for Autonomous Underwater Vehicles (AUV) in structured underwater environments. It uses a Doppler Velocity Log (DVL) sensor, a pressure sensor, a compass and a mechanically scanning imaging sonar. An Extended Kalman Filter (EKF) merges the information from the DVL and the compass to estimate the robot trajectory. In order to reduce the drift inherent to this process we use acoustic images from an imaging sonar to determine the absolute robot position in the environment and update the estimated trajectory. Copyright (C) 2007 IFAC
\end{abstract}

Keywords: Autonomous mobile robots, Kalman filters, positioning systems.

\section{INTRODUCTION}

This paper addresses the localization problem of an AUV in a known structured environment. Solving the navigation problem for an AUV which moves in a water tank opens the door to further advanced control experiments in lab conditions. Different research teams with underwater robotic testbeds, have addressed this problem in the past. In the Autonomous System Laboratory of the University of Hawaii, $\mathrm{X}$ and $\mathrm{Y}$ range measurements gathered with two fixed-bearing sonar beams are used to update a Kalman Filter (Nie, et al. 1998). Using this system, and thanks to its omni-directionality, ODIN AUV can navigate keeping its relative orientation with respect to the walls of the water tank. A more elaborated system is described in (Caccia, et al. 2001) where a profiling sonar is used to track the walls of the water tank and hence the robot is allowed to freely change its heading. In previous works, our team solved this problem using a coded pattern, lying on the bottom of a water tank, together with a real-time vision system able to provide accurate absolute position estimates at $12 \mathrm{~Hz}$ (Carreras, et al., 2003).
Further, to take part in the SAUC-E AUV competition, two new algorithms were developed. The first one used an EKF together with a simple constant velocity kinematics model, readings from a DVL sensor and a compass to compute the vehicle trajectory. In order to reduce the drift inherent to this process, an imaging sonar and an a priori map (defined as a set of lines) were used. In short, for each single beam, a high intensity point which should correspond to one object from the map is determined. After a point-to-line association process, the discrepancy among them was used to update the vehicle position (Ribas, et al., 2006). This method requires to be initialised with the vehicle position within the map, which had to be previously computed using a complete $360^{\circ}$ imaging sonar scan of the environment. Another problem arise when data association fails and the correction is computed with respect to the wrong element (line) of the a priori map, then the vehicle gets lost and the only way to be relocalized is to initialise the method again. The second developed algorithm was the one finally implemented for the competition. Basically, it consisted in an adapted version of the Hough Transform which used a complete $360^{\circ}$ imaging sonar scan and a compass to vote for the vehicle pose 
(a)

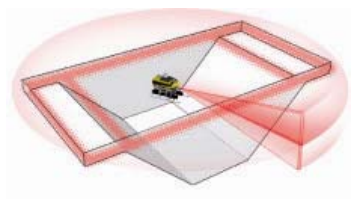

(b)

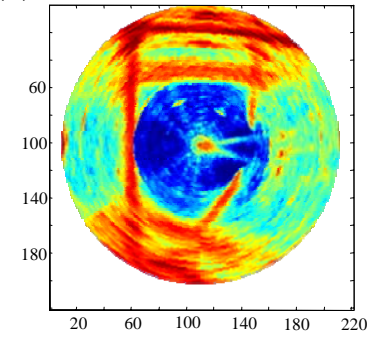

(c)

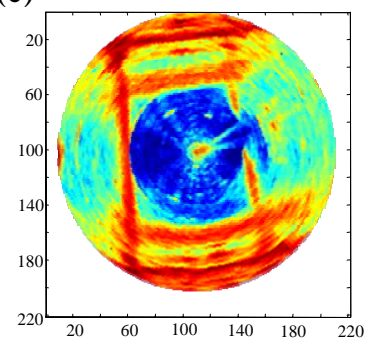

(d)

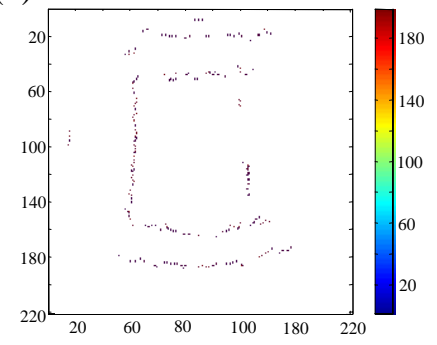

Fig. 1. (a) Schematic representation of the water tank in the Underwater Robotics Research Centre at the University of Girona where the sonar data were gathered. The highlighted zones represent the expected sonar returns. Images generated from acoustic data: (b) distorted image, (c) undistorted image after integrating vehicle position, (d) bins selected from the thresholded image to vote in our Hough space.

in a discretized space representing the environment (Ribas, et al., 2007). Its simplicity and the fact that it produced absolute position measurements were its main advantages. On the other hand, in order to fulfil the real time requirements, the cost of the voting process forced to reduce the rate at which the measurements were produced as well as its resolution.

The algorithm presented in this paper takes the best from these two algorithms. An EKF is used to continuously estimate the vehicle pose by merging the information from the different sensors. Simultaneously, a votation process is carried out to provide periodic, drift free, corrections with absolute position measurements. The advantages of the method are threefold: First, the EKF estimates the state of the vehicle during the time gap between votations, reducing the rate at which those position fixes need to be produced. Secondly, the estimated vehicle motion is used to reduce the movementinduced distortions of the acoustic images, which results in an improved votation process. Finally, obtaining absolute position fixes instead of relative ones, gets rid of the accumulating drift and avoids the vehicle getting lost.

This paper is structured as follows: Section 2 explains a method to obtain the robot position within a known environment using a voting procedure and the measurements from a mechanically scanning imaging sonar. Section 3 explains the EKF used to estimate the robot trajectory by merging the position fixes and all the sensor data. Finally, sections 4 and 5 present experimental results and conclusions.

\section{DETERMINING POSITION WITH AN IMAGING SONAR}

Mechanically scanning imaging sonar (MSIS) perform scans in a 2D plane by rotating a sonar beam through a series of small angle steps. For each emitted beam, distance vs. echo-amplitude data (also called bins) is returned forming an acoustic image of the environment (Figure 1). MSIS's usually need several seconds to obtain a complete scan (e.g. the one used in this work needs 6-20 seconds depending on the range configuration). If the vehicle is not stationary during the acquisition of the measurements, a movement induced distortion appears in the resulting acoustic data (Figure 1.b). However an undistorted scan can be obtained if vehicle displacements are taken into account (Figure 1.c). The objects present in the environment (water tank walls) appear as high echo-amplitude returns. Therefore, we can determine a threshold below which data can be discarded without loss of information. Moreover, selecting only some local (a)

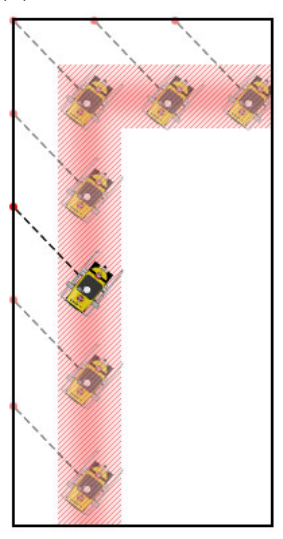

(b)

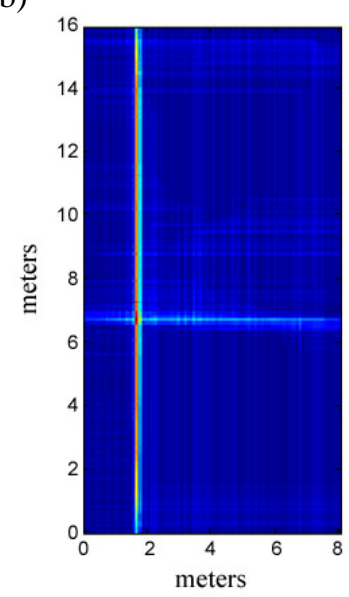

Fig. 2. (a) Diagram which represents the zone with all possible positions for the robot using a single bin from the acoustic image, (b) resulting Hough voting space for all the bins from a $360^{\circ}$ scan.

maximum among the thresholded bins reduce the number of involved measurements and hence, the computational cost (Figure 1.d).

In order to determine the robot pose we use a voting procedure which, in fact, is an adapted version of the Hough transform. The first step of the procedure is to define a discretized space representing the environment (tessellation of the water tank area). The resolution of this discretized space is a key issue in the implementation of this method as it affects the performance and the computational cost of the algorithm. A good approach is choosing a grid resolution slightly smaller than the actual resolution of the acoustic images generated by the MSIS. 
Next, the thresholded high intensity bins from a complete $360^{\circ}$ scan are used to produce votes for the robot position. Assuming that both environment and vehicle orientation are known, we can define for each single bin the locus of all the compatible vehicle positions (Figure 2.a) that should receive the votes in the Hough space. After accumulating the votes from all the bins in the voting space (Figure 2.b) the real vehicle position $\left(x_{\mathrm{MK}}, y_{\mathrm{MK}}\right)$ will correspond with the cell with the highest number of votes (i.e. the cell which accumulates more evidence about the real position of the vehicle).

The process, however, can be improved by doing some arrangements during the voting. The bins that will vote in the space are accumulated by means of a data buffer so, at any moment, we can recall information from the most recent $360^{\circ}$ scan and produce a new votation to determine the actual position of the vehicle. As new areas are explored with each beam arrival, new bins are added to the data buffer. Simultaneously, older bins falling outside the most recent $360^{\circ}$ scan sector can be removed. It is worth noting that each bin in the buffer is tagged with the position of the vehicle at the moment were the sensing took place. These positions are obtained from the EKF that is running simultaneously as explained in section 3 . When we want to produce a votation, all these positions are used to determine the location of all the stored bins with respect to the last (actual) vehicle position. At this point, what we obtain is a set of measurements that are similar to those we would obtain if a sensor, placed in the actual vehicle position, took a snapshot of the scene. Voting with this corrected set of bins has two effects. First, as we take into account the relative vehicle positions, the voting is done with undistorted data. Second, as we have referenced all the bins with respect to the current vehicle position, the votes will fall within this position instead of letting them fall all along the vehicle trajectory.

\section{EKF BASED LOCALIZATION SYSTEM}

An EKF is used to estimate the vehicle position by merging the measurements from a DVL, a pressure sensor and a compass. Due to the integration of the velocity measurements the estimated trajectory will drift. To avoid this effect, we use the absolute position fixes obtained from the voting algorithm presented in section 2 to correct the current position.

The estimate of the vehicle state $\mathbf{x}$ is represented as:

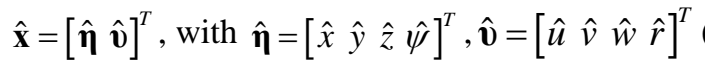

$$
\begin{aligned}
& \mathbf{P}=E\left(\left[\mathbf{x}_{k}-\hat{\mathbf{x}}_{k}\right]\left[\mathbf{x}_{k}-\hat{\mathbf{x}}_{k}\right]^{T} \mid \mathbf{Z}\right)
\end{aligned}
$$

where, as defined in (Fossen, 2002), $\boldsymbol{\eta}$ is the position an attitude vector of the vehicle while $\boldsymbol{v}$ is its linear and angular velocity vector. Note that although the vehicle position is represented in a global base frame, the velocities in $\mathbf{v}$ are represented in the vehicle coordinate frame. The position of the global base frame can be set arbitrarily. However, for simplicity we chose one of the corners of the water tank.

\subsection{Initialization.}

In order to initialize the filter, we gather a complete $360^{\circ}$ imaging sonar scan and then the voting algorithm is used to determine the initial position of the vehicle. We assume that at the beginning of the experiment, the velocity of the vehicle is almost zero and thus, the displacement can be neglected. The values in the diagonal of $\mathbf{P}$ should be initialized with the expected accuracy of the position measurements.

\subsection{Prediction.}

A simple 4DOF constant velocity kinematics model is used to predict the state of the vehicle. Since AUVs are commonly operated describing rectilinear transects at constant speed, we believe that such model is a simple but realistic way to describe the motion.

$$
\hat{\mathbf{x}}_{k+1}^{-}=\left[\begin{array}{c}
x \\
y \\
z \\
\psi \\
u \\
v \\
w \\
r
\end{array}\right]_{k+1}=\left[\begin{array}{c}
x_{k}+u_{k} t \cos \left(\psi_{k}\right)-v_{k} t \sin \left(\psi_{k}\right) \\
y_{k}+u_{k} t \sin \left(\psi_{k}\right)+v_{k} t \cos \left(\psi_{k}\right) \\
z_{k}+w_{k} t \\
\psi_{k}+r_{k} t \\
u_{k} \\
v_{k} \\
w_{k} \\
r_{k}
\end{array}\right]
$$

The unmodelled perturbations in the model are represented by an additive zero mean white Gaussian noise with covariance $\mathbf{Q}$ affecting the velocity terms and propagated to the position through integration.

The state uncertainty is also predicted using the typical EKF equations:

$$
\mathbf{P}_{k+1}^{-}=\mathbf{F}_{k} \mathbf{P}_{k} \mathbf{F}_{k}^{T}+\mathbf{G}_{k} \mathbf{Q}_{k} \mathbf{G}_{k}^{T}
$$

where $\mathbf{F}$ and $\mathbf{G}$ are the Jacobian matrices of partial derivatives of the model with respect to the state $\mathbf{x}$ and the noise respectively.

$\mathbf{F}=\left[\begin{array}{cccccccc}1 & 0 & 0 & -u t \sin (\psi)-v t \cos (\psi) & t \cos (\psi) & -t \sin (\psi) & 0 & 0 \\ 0 & 1 & 0 & u t \cos (\psi)-v t \sin (\psi) & t \sin (\psi) & t \cos (\psi) & 0 & 0 \\ 0 & 0 & 1 & 0 & 0 & 0 & t & 0 \\ 0 & 0 & 0 & 1 & 0 & 0 & 0 & t \\ 0 & 0 & 0 & 0 & 1 & 0 & 0 & 0 \\ 0 & 0 & 0 & 0 & 0 & 1 & 0 & 0 \\ 0 & 0 & 0 & 0 & 0 & 0 & 1 & 0 \\ 0 & 0 & 0 & 0 & 0 & 0 & 0 & 0\end{array}\right]$ (5)




$$
\mathbf{G}=\left[\begin{array}{cccc}
\frac{t^{2}}{2} \cos (\psi) & -\frac{t^{2}}{2} \sin (\psi) & 0 & 0 \\
\frac{t^{2}}{2} \sin (\psi) & \frac{t^{2}}{2} \cos (\psi) & \frac{t^{2}}{2} & 0 \\
0 & 0 & 0 & \frac{t^{2}}{2} \\
0 & 0 & 0 & 0 \\
t & 0 & 0 & 0 \\
0 & t & 0 & 0 \\
0 & 0 & t & 0 \\
0 & 0 & 0 & t
\end{array}\right]
$$

\subsection{DVL update.}

A SonTek Argonaut DVL unit provides bottom tracking and water velocity measurements but also includes a pressure sensor for depth estimation. Hence, the measurement vector can be described as:

$$
\mathbf{z}_{D V L}=\left[\begin{array}{lllllll}
z_{\text {depth }} & u_{w} & v_{w} & w_{w} & u_{b} & v_{b} & w_{b}
\end{array}\right]^{T}
$$

where subindex $b$ stands for bottom tracking velocity and $w$ for through water velocity. Note that we assume that no water currents are present in the application scenario, therefore through water velocity measurements can be used to estimate the vehicle velocities. As all those measurements are direct observation of the state vector we can use a linear measurement model as:

$$
\mathbf{z}_{D V L, k}=\mathbf{H}_{D V L} \hat{\mathbf{x}}_{k+1}^{-}+\mathbf{s}_{D V L}
$$

with

$$
\mathbf{H}_{D V L}=\left[\begin{array}{cccc}
0_{1 \times 2} & 1 & 0_{1 \times 3} & 0_{1 \times 2} \\
0_{3 x 2} & 0_{3 x 2} & I_{3 \times 3} & 0_{3 x 1} \\
0_{3 \times 2} & 0_{3 \times 2} & I_{3 \times 3} & 0_{3 \times 1}
\end{array}\right]
$$

where $\mathbf{s}_{D V L}$ (measurement noise) is a zero-mean white noise with covariance $\mathbf{R}_{D V L}$.

Since the DVL sensor provides a status measurement for the bottom tracking and water velocity, depending on the quality of the measurements, different versions of the $\mathbf{H}$ matrix are used to fuse one (removing row 2), the other one (removing row 3 ), or both readings (using the full matrix).

The update can be done with the standard KF equations.

$$
\begin{aligned}
& K_{k}=\mathbf{P}_{k+1}^{-} \mathbf{H}_{D V L}^{T}\left(\mathbf{H}_{D V L} \mathbf{P}_{k+1}^{-} \mathbf{H}_{D V L}^{T}+\mathbf{R}_{D V L}\right)^{-1} \\
& \hat{\mathbf{x}}_{k+1}=\hat{\mathbf{x}}_{k+1}^{-}+K_{k}\left(\mathbf{z}_{D V L, k}-\mathbf{H}_{D V L} \hat{\mathbf{x}}_{k+1}^{-}\right)
\end{aligned}
$$

$$
\mathbf{P}_{k+1}=\left(\mathbf{I}-K_{k} \mathbf{H}_{D V L}\right) \mathbf{P}_{k+1}^{-}
$$

\subsection{Compass update.}

A MRU MTi from Xsens Technologies provides information about the heading of the vehicle. Its measurement is described as:

$$
\mathbf{z}_{C}=\left[\psi_{C}\right]
$$

As this measurement is a direct observation of the state vector, we can use the following linear measurement model.

$$
\mathbf{z}_{C, k}=\mathbf{H}_{C} \hat{\mathbf{x}}_{k+1}^{-}+\mathbf{s}_{C}
$$

with

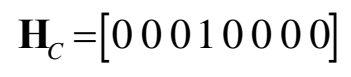

where $\mathbf{s}_{C}$ (measurement noise) is a zero-mean white noise with covariance $\mathbf{R}_{C}$.

The standard KF equations are used to update the state:

$$
\begin{gathered}
K_{k}=\mathbf{P}_{k+1}^{-} \mathbf{H}_{C}^{T}\left(\mathbf{H}_{C} \mathbf{P}_{k+1}^{-} \mathbf{H}_{C}^{T}+\mathbf{R}_{C}\right)^{-1} \\
\hat{\mathbf{x}}_{k+1}=\hat{\mathbf{x}}_{k+1}^{-}+K_{k}\left(\mathbf{z}_{C, k}-\mathbf{H}_{C} \hat{\mathbf{x}}_{k+1}^{-}\right) \\
\mathbf{P}_{k+1}=\left(\mathbf{I}-K_{k} \mathbf{H}_{C}\right) \mathbf{P}_{k+1}^{-}
\end{gathered}
$$

\subsection{Sonar beam arrival.}

As introduced in section 2, whenever a new beam is produced, a search for the high intensity bins is carried out and then, they are stored in the buffer together with its current position estimate from $\hat{\mathbf{x}}^{-}$.

Periodically, the information from the data buffer is recalled to produce a votation with the bins from the most recent $360^{\circ}$ scan (in our particular application a votation is performed each 25 beams). The output of this voting process is an estimate of the absolute vehicle position within the environment $\left(x_{\mathrm{M \kappa}}, y_{\mathrm{MK}}\right)$. Therefore, we can perform an update that will reduce the accumulated drift as:

$$
\begin{gathered}
\mathbf{z}_{M K}=\left[\begin{array}{ll}
x_{M K} & y_{M K}
\end{array}\right]^{T} \\
\mathbf{z}_{M K, k}=\mathbf{H}_{M K} \hat{\mathbf{x}}_{k+1}^{-}+S_{M K} \\
\mathbf{H}_{M K}=\left[\begin{array}{ll}
\mathbf{I}_{2 \times 2} & 0_{2 \times 6}
\end{array}\right]
\end{gathered}
$$


where $\mathbf{s}_{M K}$ (measurement noise) is a zero-mean white noise with covariance $\mathbf{R}_{M K}$. It is worth noting that the measured absolute position obtained from the voting process is not strictly a gaussian measurement because of the discretization of the voting space. However, we believe that assuming a pessimistic uncertainty description of the measurement copes with the induced non-gaussianities. The results presented in section 4 confirm the validity of this statement.

Again, the update is done with the standard KF equations:

$$
\begin{gathered}
K_{k}=\mathbf{P}_{k+1}^{-} \mathbf{H}_{M K}^{T}\left(\mathbf{H}_{M K} \mathbf{P}_{k+1}^{-} \mathbf{H}_{M K}^{T}+\mathbf{R}_{M K}\right)^{-1} \\
\hat{\mathbf{x}}_{k+1}=\hat{\mathbf{x}}_{k+1}^{-}+K_{k}\left(\mathbf{z}_{M K, k}-\mathbf{H}_{M K} \hat{\mathbf{x}}_{k+1}^{-}\right) \\
\mathbf{P}_{k+1}=\left(\mathbf{I}-K_{k} \mathbf{H}_{M K}\right) \mathbf{P}_{k+1}^{-}
\end{gathered}
$$

As this is an absolute measurement, taken with respect to the walls of the water tank, the performed update will reduce the accumulated drift.

\section{EXPERIMENTAL RESULTS}

Experiments were carried out in the water tank of the Underwater Robotics Research Centre at the University of Girona (Figure 3). The ICTINEU ${ }^{\text {AUV }}$ was used as testbed for these experiments. The vehicle was equipped with a Miniking Imaging sonar from Tritech, which during the experiment was set to work within a range of $18 \mathrm{~m}$ and a resolution of $0.1 \mathrm{~m}$ (180 measurements/beam). Its scanning rate was set to the maximum (around 6 seconds for a $360^{\circ}$ scan with 200 beams). In order to estimate the vehicle movement an Argonaut DVL from Sontek and a MRU MTi from Xsens Technologies were used.
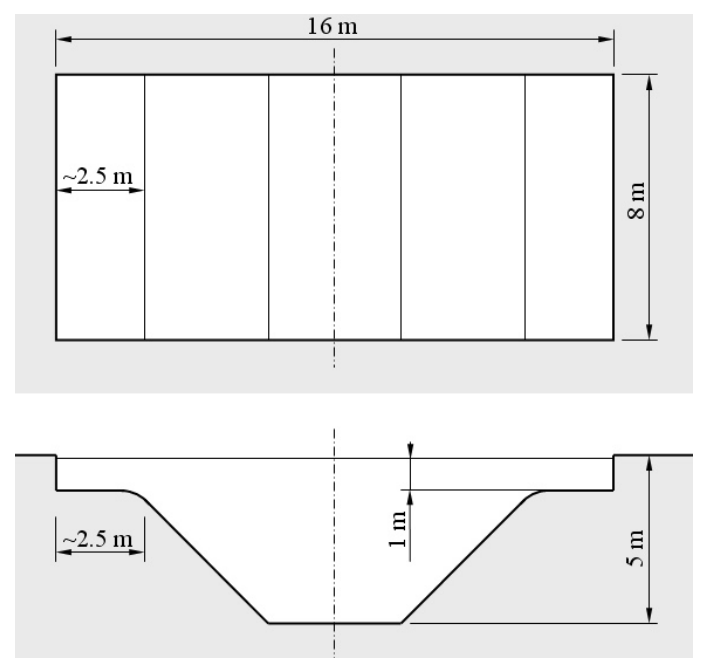

Fig. 3. Representation of the water tank at the Underwater Robotics Research Centre (University of Girona) were the experiments took place.
Two experiments have been carried out to check the algorithm. In the first one, the vehicle was operated near the surface in the deep part of the water tank (about 5 meters) to describe a square-shaped trajectory which ended with a diagonal transect. The second one was a similar trajectory, but this time, the vehicle also moved over the shallow zones of the tank.

It is important to mention that the DVL sensor needs a minimum water volume to provide measurements. In the shallow parts of the tank, or when the vehicle is navigating near the bottom or the walls, no velocity measurements are available. Thus, the estimate only relies on the kinematics model and the fixes from the voting algorithm. The purpose of these experiments was to test the reliability of the algorithm in different working conditions.

In Figure 4, the first experiment is shown. The trajectory represented with a black-dotted line corresponds to a dead reckoning obtained using only the measurements from the DVL and the compass. As expected in any dead-reckoning trajectory, it can be observed a significant drift which brings the vehicle out of the limits of the water tank (represented by a thick black line). Moreover, this drift is also increased because the measurements from the two sensors are affected by the proximity of the tank walls. On the other hand, using the absolute position fixes from the voting algorithm (blue dots), conveniently merged with the estimate from the EKF results in a more accurate trajectory without drift (in black).

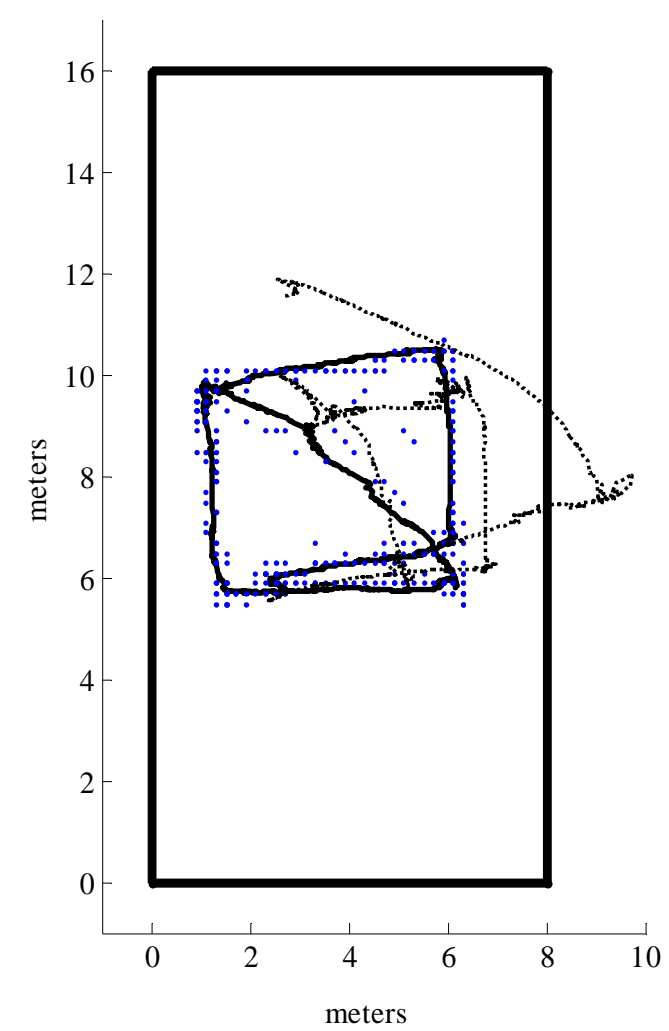

Fig. 4. Resulting trajectory for the first experiment (black) after merging the position estimates from acoustic images (dots) with the dead reckoning trajectory (dotted line) with an EKF. 
Results for the second experiment can be observed in Figure 5. As commented before, we should expect a worse dead reckoning trajectory (dotted line) as the DVL do not work properly in the shallow zones of the tank. However, the proposed algorithm again is able to relocalize the vehicle into drift-free trajectory estimation.

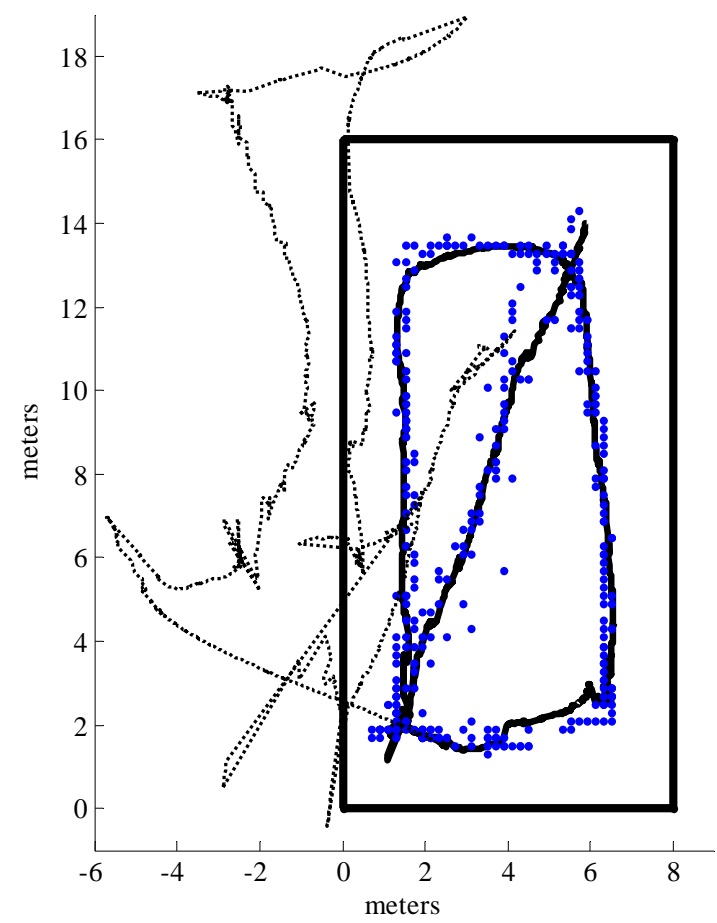

Fig. 5. Resulting trajectory for the second experiment (black) after merging the position estimates from acoustic images (dots) with the dead reckoning trajectory (dotted line) with an EKF.

If we take a look at the evolution of the uncertainty of the dead reckoning and the proposed process (Figure 6) we can observe how the dead reckoning trajectory suffers from an unbounded uncertainty growth (dotted line) while the integration of the position fixes reduces and bounds its value (in black).

\section{CONCLUSIONS}

In this paper we have presented an improved version of the localization algorithms proposed for the SAUC-E competition. An EKF based algorithm which relies in the output from traditional underwater navigation sensors together with position fixes from an adapted Hough transform voting process is presented. There are two enhancements as a result of the proposed method. First, we can get rid of the drift inherent in dead reckoning sensors. Second, an improved estimation of the vehicle movement can be obtained as we can rely in shortterm estimation from the EKF during the time gaps between absolute position fixes.
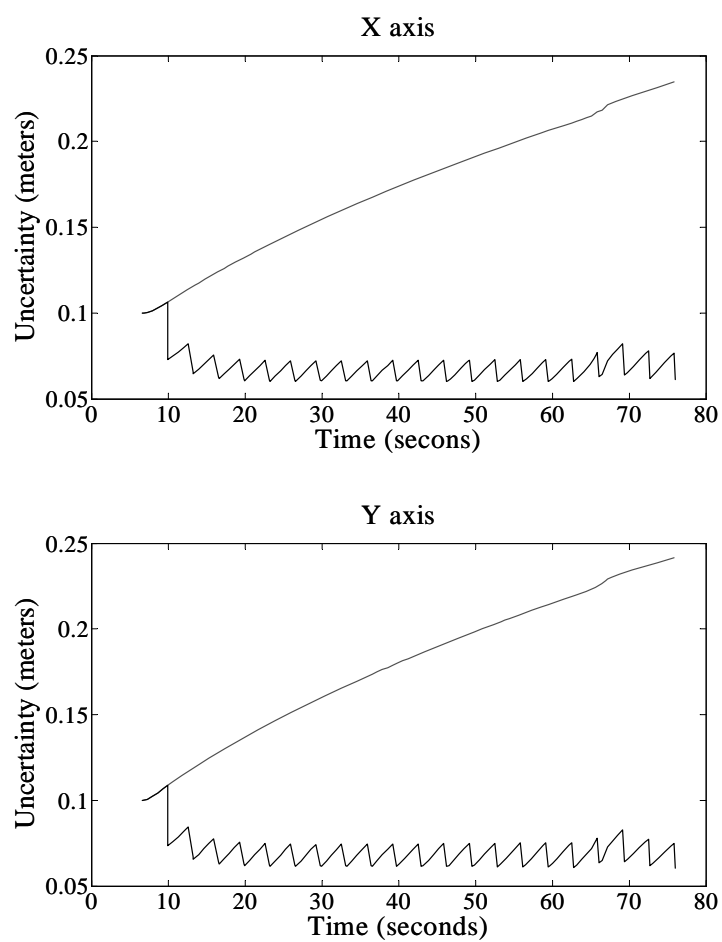

Fig. 6. Evolution of the uncertainty in $\mathrm{x}$ and $\mathrm{y}$ axis for the dead reckoning trajectory (dotted line) and the proposed evolution (black) during the first 80 seconds of the first experiment.

\section{REFERENCES}

Caccia, M., Bruzzone, G. And Veruggio G. (2001). Sonar-based guidance of unmanned underwater vehicles. Advanced robotics, Vol. 15, No.5, pp.551-573

Carreras, M., Ridao, P., Garcia, R. and Nicosevici, T. (2003). Vision-based Localization of an Underwater Robot in a Structured Environment. International Conference on Robotics and Automation.

Fossen, T. (2002). Marine Control Systems. Guidance, Navigation, and Control of Ships, Rigs and Underwater Vehicles. Marine Cybernetics AS.

Nie, J., Yuh, J., Kardash, E. and Fossen, T. (1998). On-Board Sensor-Based Adaptive Control Of Small Uuvs In Very Shallow Water. Control Applications in Marina Systems.

Ribas, D., Palomeras, N., Ridao, P., Carreras, M. and Hernandez, E. (2007). ICTINEU AUV Wins the first SAUC-E competition. International Conference on Robotics and Automation.

Ribas, D., Neira, J., Ridao, P. and Tardos, J.D. (2006). AUV localization in structured underwater environments using an a priori map. Manoeuvring and Control of Marine Crafts. 